\title{
BMJ Open Effectiveness and feasibility of internet- based and mobile-based interventions for individuals experiencing bereavement: a systematic review protocol
}

\author{
Melanie Luppa (D) , Margrit Löbner, Alexander Pabst, Christine Schlapke, \\ Janine Stein, Steffi G Riedel-Heller
}

To cite: Luppa M, Löbner M, Pabst A, et al. Effectiveness and feasibility of internet-based and mobile-based interventions for individuals experiencing bereavement: a systematic review protocol. BMJ Open 2020;10:e036034. doi:10.1136/ bmjopen-2019-036034

- Prepublication history and additional material for this paper are available online. To view these files, please visit the journal online (http://dx.doi. org/10.1136/bmjopen-2019036034).

Received 02 December 2019 Revised 28 February 2020 Accepted 20 March 2020

\section{Check for updates}

(C) Author(s) (or their employer(s)) 2020. Re-use permitted under CC BY-NC. No commercial re-use. See rights and permissions. Published by BMJ.

Institute of Social Medicine, Occupational Health and Public Health, University of Leipzig, Faculty of Medicine, Leipzig, Sachsen, Germany

Correspondence to Dr Melanie Luppa;

Melanie.Luppa@medizin.unileipzig.de

\section{ABSTRACT}

Introduction Internet-based and mobile-based interventions (IMIs) provide an innovative and efficient self-management tool for mental health problems. This systematic review aims to summarise and critically evaluate studies addressing the effectiveness and feasibility of IMls for normal and complicated grief in bereaved adults.

Methods and analysis The databases MEDLINE, Cochrane Library, PsycINF0, Embase and Web of Science and Google Scholar (for 'grey' literature) will be systematically searched for feasibility studies or randomised controlled trials of IMls for bereaved adults who were experiencing normal/complicated grief. Data will be extracted and evaluated independently by two reviewers from studies eligible for inclusion. Quality of evidence will be assessed, and results will be synthesised qualitatively and pooled meta-analytically, if sufficient outcome data are available. Preferred Reporting Items for Systematic Reviews and Meta-Analyses standards and Grades of Recommendation, Assessment, Development and Evaluation methodology will be used.

Ethics and dissemination No primary data will be collected; thus, ethical approval is not required. The results will be disseminated through a peer-reviewed publication and conference presentations.

Trial registration number CRD42019131428.

\section{BACKGROUND}

Self-management is a widely used approach within the medical healthcare system for improving patients' knowledge, capabilities and skills in managing their health problems. Internet-based and mobile-based interventions (IMIs) provide an innovative and efficient self-management tool for mental health problems. In recent years, web-based self-management interventions have gained increasing attention as effective supplementary treatment elements to standard mental health treatment. ${ }^{1}$
Strengths and limitations of this study

First study that provides a comprehensive summary of studies investigating effectiveness and feasibility of internet-based and mobile-based interventions (IMIs) for normal and complicated grief in bereaved adults.

- Possibility of usage of these IMls in healthcare as additional intervention tools as well as low-threshold treatment options.

- Application with the necessary caution of these IMIs seems to be required not to interfere with natural grief processes.

The effectiveness of IMIs has been shown for treating depression (eg, refs 2-4), anxiety (eg, refs 5 6), post-traumatic stress disorder (PTSD; eg, refs 7-9) and other mental health problems. ${ }^{1}$ However, less is currently known about IMIs for individuals experiencing normal or complicated grief.

In general, grief is defined as a typical reaction to the loss of a significant other ${ }^{10}$ and is associated with symptoms such as intense subjective distress, loneliness and somatic symptoms, for example, tightness of the throat or need for sighing. ${ }^{11} 12$ Recently, the concept of an abnormal reaction to loss has been proposed and is included as a disorder in the Diagnostic Statistical Manual of Mental Disorders, Fifth Edition, and is expected to be included in the International Statistical Classification of Diseases and Related Health Problems, 11th Revision as a new diagnosis. The disorders complicated, traumatic or prolonged grief (subsequently summarised under complicated grief) and complicated grief are described as 'a syndrome of prolonged and intense grief that is accompanied by complications that derail the progress of grief'. ${ }^{13}$ 


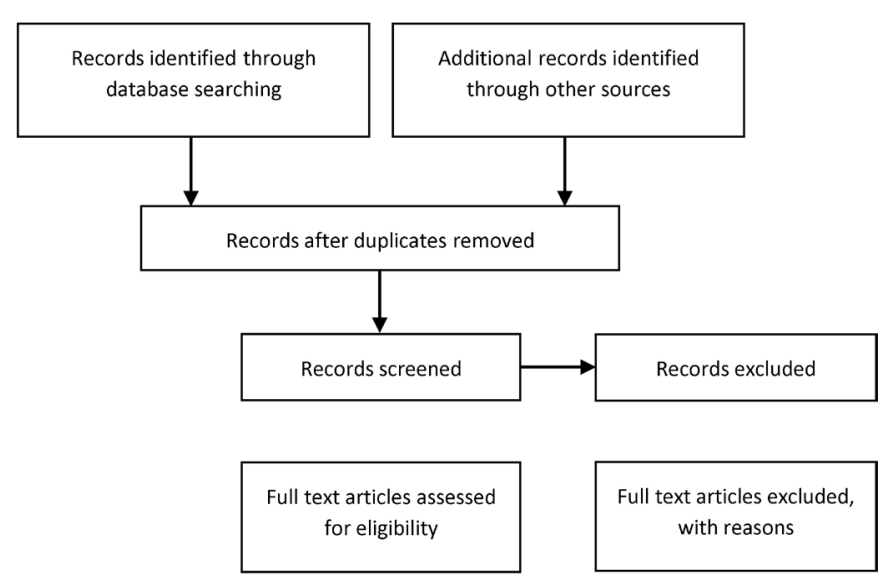

Studies included in

methodological and

quantitative evaluation

Figure 1 Flow diagram of the planned study selection process adapted from the Preferred Reporting Items for Systematic Reviews and Meta-Analyses statement.

In contrast to uncomplicated grief, clinically significant impairment in social, occupational or other important areas of functioning must be present. ${ }^{13} 14$ The diagnosis of complicated grief is given only after a period of 6 months following the loss event if the person is still suffering from separation stress as well as cognitive, emotional and behavioural symptoms. ${ }^{14}$

Because grief can affect many areas of life $\mathrm{e}^{1516}$ and is one of the major contributors to the development of mental health disorders, providing IMIs as a low-threshold treatment option may enable more people to receive treatment than through face-to-face interventions alone ${ }^{17}$ to prevent the development of mental health disorders. Major depression, generalised anxiety disorder and PTSD are particularly closely related to the symptoms of complicated grief, but differences between the disorders have also been reported. ${ }^{1418}$

To date, a number of IMIs for bereavement problems exist in the international research literature. Internetbased cognitive-behavioural therapy (CBT) interventions were reported to be effective in treating patients with complicated grief ${ }^{19}$ but not for those with uncomplicated grief. ${ }^{20}$ Some researchers have urged caution in implementing interventions too early or across a wide range of bereavement-related distress so as to not interfere with natural grief processes. ${ }^{21} 22$ One study showed that IMIs for PTSD improve symptoms of complicated grief. ${ }^{23}$ To our knowledge, there are no previous systematic reviews summarising the effectiveness and feasibility of IMIs for bereaved individuals-for normal as well as complicated grief. The results of this review and meta-analysis will therefore address this gap in the literature. This protocol describes the rationale and design of the planned systematic review and meta-analysis.

\section{OBJECTIVES}

The planned review aims to systematically evaluate and synthesise the evidence base of randomised controlled trials (RCTs) reporting, the effectiveness of IMIs (ie, improvement of objective parameters) and the feasibility of IMIs (eg, usability, satisfaction, acceptability, understandability and usefulness) for individuals aged 18 years and older who experienced the death of a significant other. Depending on the number of eligible studies, assessment tools and quality of the studies reported, we will also combine data across RCTs to estimate pooled effect sizes for the considered outcomes.

\section{METHODS AND ANALYSIS}

This protocol outlines the strategies for conducting a systematic review and meta-analysis of RCTs that examined the effectiveness of IMIs for bereavement. It is based on the Preferred Reporting Items for Systematic Reviews and Meta-Analyses (PRISMA) for systematic review protocols (PRISMA-P) guidelines. ${ }^{24}{ }^{25}$ The protocol describes the planned strategy to systematically evaluate and synthesise data from RCTs and feasibility studies on IMIs for bereaved individuals. We will apply the four-phase PRISMA flow diagram (figure 1) for our study selection process.

\section{Eligibility criteria}

The systematic review will be divided into two parts: effectiveness studies and feasibility studies on IMIs (study design criteria) that include adults (18 years and older) who experienced the death of a significant other and were suffering from normal or complicated grief (participant criteria). Any measures of effectiveness (ie, improvement of objective parameters) and feasibility (eg, usability, satisfaction, acceptability, understandability and usefulness) (outcome criteria) of IMIs related to bereavement will be included. Onset data from clinician-rated scales will be prioritised over self-report questionnaires. The intervention must have been a psychological intervention according to Kampling et $a l^{26}$ criteria: CBT, psychodynamic psychotherapy, behaviour therapy or behaviour modification, systemic therapy, third wave CBT, humanistic therapy, integrative therapy or to other psychologicalorientated interventions and must have been provided in an online setting (intervention criteria). In RCTs, the comparison group must be either 'treatment as usual', 'waiting list', 'attention placebo' (inactivity on the part of both researchers and participants) or 'psychological placebo' (activity on the part of participants and inactivity of researchers). We will consider articles that are written in either English or German (language criteria). The literature search will not be restricted by publication date.

\section{Information sources and search strategy}

Systematic literature searches will be conducted in the databases MEDLINE (PubMed interface), Cochrane Library (Cochrane Database of Systematic Reviews, Cochrane 
Central Register of Controlled Trials (CENTRAL) and Cochrane Methodology Register), PsycINFO, Embase, Web of Science (Science and Social Science Citation Index) and Google Scholar (for 'grey' literature) by MeL and CS independently. A combination of the following search terms will be used: (1) bereavement or widowhood or grief; and (2) online or web or computer or mobile or e-health or internet; and (3) intervention or psychotherapy or cognitive behavioural therapy or CBT. The draft of the full MEDLINE search strategy is available in online supplementary appendix 1. If feasible, medical subject headings will be used as search terms. The finalised MEDLINE search strategy will be adapted to the syntax and subject headings specifications of the other databases. We will initially screen titles and abstracts for eligibility. Full texts will then be assessed for criteria, and the reference lists of included articles and systematic reviews will be hand searched to identify further potentially relevant studies. Finally, we will conduct a grey literature search for unpublished studies using Google and Google Scholar with the above-named search terms. If applicable and necessary, we will contact researchers directly to gather further relevant non-published data. The searches will be rerun just before the final analyses so that more recent studies can be retrieved.

\section{Data management}

References and data will be managed using the Review Manager (RevMan) software package V.5.3 (by the Nordic Cochrane Centre, The Cochrane Collaboration, Copenhagen, Denmark, 2014). RevMan is specifically designed for managing and analysing systematic review data from bibliographical management to data synthesis. If feasible, additional data analyses and meta-analysis will be conducted using Stata V.13.1 SE.

\section{Selection process}

All titles and abstracts of articles will be screened independently by two reviewers (MeL and CS). At this stage, articles will be divided into potentially relevant, irrelevant or uncertain. Reasons for exclusion of irrelevant articles will be given. Potentially relevant and uncertain articles will be read in full text independently by MeL and CS, and study eligibility based on the established criteria specified above will be assessed. At each stage of the selection process, any discrepancies will be discussed between the two reviewers. When discrepancies cannot be resolved, input from a third senior researcher (SGR-H) will be obtained.

\section{Data collection process and data items}

A standardised data extraction form will be used to extract data from included studies. Extracted data will include study characteristics, participant characteristics, methodological factors and outcome data. A pilot version of the data extraction form will be tested independently by the two reviewers (MeL and CS) on a subsample of relevant studies to ensure correct extraction of all relevant data. Difficulties with data extraction will be discussed and the form will be adopted accordingly. Data from each study will be extracted by both reviewers (MeL and CS) independently, and reliability of data extraction will be checked in a random sample of studies. Discrepancies between the two reviewers will be discussed with a senior researcher (SGR-H). Missing data will be requested from study authors.

The following data will be extracted:

1. Study identification items: for example, first author, year of publication and country.

2. Study design characteristics: for example, sample size, recruitment strategy, inclusion/exclusion criteria, circumstances of the loss (eg, violent death and suicide), control group, diagnostic criteria/assessment of nor$\mathrm{mal} / \mathrm{prolonged} / \mathrm{complicated}$ grief, assessment of cooccurring conditions (eg, Major depression, PTSD and concurrent pharmacotherapy/psychotherapy), assessment of suicidal ideation or behaviour, interventions design/type, duration of intervention and length of follow-up assessments.

3. Participants characteristics: for example, mean age, age range and gender.

4. Methodological aspects: risk of bias and study limitations.

5. Outcomes: (A) effectiveness: primary outcome measures: reduction of grief symptoms; secondary outcome measures: reduction of depression, anxiety, somatisation or PTS symptoms or suicidal ideation or behaviour and (B) feasibility: usability, satisfaction, acceptability, understandability and usefulness; onset data from clinician-rated scales will be prioritised over self-report questionnaires. All different time frames of follow-up assessments will be included.

\section{Quality assessment}

The methodological quality of included studies will be assessed by two researchers (MeL and CS) independently using the Cochrane Collaboration Tool for Assessing Risk of Bias in RCTs. ${ }^{27}$ As recommended, each study will be assessed in the following domains: (1) selection bias, that is, descriptions of the (1a) method of randomisation and (1b) concealment of allocation; (2) performance bias, that is, description of the methods of blinding participants and researchers; (3) detection bias, that is, description of the methods of blinding outcome assessment; (4) attrition bias, that is, description of incomplete outcome data; (5) reporting bias, that is, description of selective outcome reporting; and (6) other bias, that is, description of important concerns about other biases. Studies will be rated as 'high', 'low' or 'unclear'. These assessments will be used to inform the corresponding Grades of Recommendation, Assessment, Development and Evaluation (GRADE) assessment of study limitations (see table 5.6 of the GRADE handbook. ${ }^{28}$ Specifically, 'low risk of bias' would indicate 'no limitation'; 'unclear risk of bias' would indicate either 'no limitation' or 'serious limitation'; and 'high risk of bias' would indicate either 
'serious limitation' or 'very serious limitation' in the GRADE approach. Any disagreement between the two reviewers will be resolved by discussions with involvement of a third review author where necessary. Study authors will be contacted for further methodological information if needed. In the risk of bias table, results of the judgements will be shown for each domain.

\section{Data synthesis and presentation}

A narrative synthesis for all included studies and relevant characteristics listed under 'data collection process' will be provided in text and 'summary of findings' tables. Characteristics of the study, sample, intervention and control condition will be presented first, followed by outcome measurements, effect sizes and overall results.

Only studies that provide a quantitative measure of grief symptoms will be included in the meta-analysis. We will analyse heterogeneity by providing $I^{2}$ statistics and funnel and forest plots. According to the Cochrane standards, we suppose a moderate level of heterogeneity between studies for $I^{2}$ values ranging from $30 \%$ to $60 \% .^{29}$ If studies fail to show sufficient heterogeneity $\left(I^{2}<60 \%\right)$ in at least two trials, ${ }^{30}$ meta-analytic pooling will not be undertaken. However, inconsistency may occur from differences in study characteristics. ${ }^{29}$ Therefore, we will explore sources of heterogeneity in subgroups of studies in terms of type of grief or intervention type. A random effects model will be applied. We will estimate standardised mean difference values and the respective $95 \%$ CIs. We will follow the Cochrane Handbook for Systematic Reviews of Interventions ${ }^{27}$ to deal with missing data.

Data analyses will be performed using RevMan V.5.3 software from the Cochrane Collaboration Tool for Implementing the Characteristics of Studies (RevMan (computer program), 2014).

\section{Patient and public involvement}

No patient and/or the public involved.

\section{DISCUSSION}

The planned systematic review will provide a comprehensive summary of the effectiveness and feasibility of IMIs for adults who suffer from bereavement.

If treatment programmes for bereavement that use either the internet or mobile phone technology show effectiveness and feasibility, this therapeutic delivery method has the potential to become an additional intervention tool. Internet-based interventions can reach more people than face-to-face interventions. ${ }^{17}$ Their costeffectiveness for depression ${ }^{31}$ and anxiety disorders ${ }^{32} 33$ have also been demonstrated. Because of a prevalence rate of $3.7 \%$ for complicated grief in Germany, ${ }^{34}$ a lowthreshold treatment option would enable the provision of adequate care to more bereaved adults.

If there are an insufficient number of studies that have examined gender and age differences, this will be discussed in terms of a need for future research. Second, this review could motivate other researchers to construct and test in randomised trials new or modified internetbased or mobile-based interventions for bereaved adults.

\section{Amendments}

In the event of protocol amendments, we will provide the date, a description of and rationale for of each amendment.

Contributors All authors contributed substantially to the conception of the work; MeL and CS drafted the manuscript; AP, MaL, JS and SGR-H revised the manuscript critically for important intellectual content; all authors finally approved the version to be published. All authors gave agreement to be accountable for all aspects of the work.

Funding This publication is part of the AgE health study and was funded by the German Federal Ministry of Education and Research (reference number: 01GY1613).

Competing interests None declared.

Patient consent for publication Not required.

Ethics approval Ethical approval and consent to participate are not required as no primary data will be collected. The results of this systematic review are intended to be published in an international peer-reviewed journal. Results may also be presented at relevant professional conferences and meetings.

Provenance and peer review Not commissioned; externally peer reviewed.

Open access This is an open access article distributed in accordance with the Creative Commons Attribution Non Commercial (CC BY-NC 4.0) license, which permits others to distribute, remix, adapt, build upon this work non-commercially, and license their derivative works on different terms, provided the original work is properly cited, appropriate credit is given, any changes made indicated, and the use is non-commercial. See: http://creativecommons.org/licenses/by-nc/4.0/.

Author note All authors approved the final version of the manuscript.

ORCID iD

Melanie Luppa http://orcid.org/0000-0003-3927-6728

\section{REFERENCES}

1 Stein J, Röhr S, Luck T, et al. Indikationen und Evidenz von international entwickelten Online-Coaches Zur intervention bei psychischen Erkrankungen - ein Meta-Review. Psychiatr Prax 2018:45:7-15.

2 Richards D, Duffy D, Burke J, et al. Supported internet-delivered cognitive behavior treatment for adults with severe depressive symptoms: a secondary analysis. JMIR Ment Health 2018;5:e10204.

3 Twomey C, O'Reilly G, Meyer B. Effectiveness of an individuallytailored computerised CBT programme (Deprexis) for depression: a meta-analysis. Psychiatry Res 2017;256:371-7.

4 Karyotaki E, Ebert DD, Donkin L, et al. Do guided Internet-based interventions result in clinically relevant changes for patients with depression? an individual participant data meta-analysis. Clin Psychol Rev 2018;63:80-92.

5 Morgan C, Mason E, Newby JM, et al. The effectiveness of unguided Internet cognitive behavioural therapy for mixed anxiety and depression. Internet Interv 2017;10:47-53.

6 Morris J, Firkins A, Millings A, et al. Internet-Delivered cognitive behavior therapy for anxiety and insomnia in a higher education context. Anxiety Stress Coping 2016;29:415-31.

7 Lange A, van de Ven J-P, Schrieken B. Interapy: treatment of posttraumatic stress via the Internet. Cogn Behav Ther 2003;32:110-24.

8 Sijbrandij M, Kunovski I, Cuijpers P. Effectiveness of internetdelivered cognitive behavioral therapy for posttraumatic stress disorder: a systematic review and meta-analysis. Depress Anxiety 2016;33:783-91.

9 Spence J, Titov N, Dear BF, et al. Randomized controlled trial of internet-delivered cognitive behavioral therapy for posttraumatic stress disorder. Depress Anxiety 2011;28:541-50.

10 Lammer K. Trauer verstehen. Formen, Erklärungen, Hilfen. Berlin: Springer, 2014. http://dx.doi.org/10.1007/978-3-642-41667-5

11 Jackson EN. Understanding grief. Pastoral Psychol 1957;8:41-8.

12 Lindemann E. Symptomatology and management of acute grief. 1944. Am J Psychiatry 1994;151:141-8. 
13 Zisook S, Simon NM, Reynolds CF, et al. Bereavement, complicated grief, and DSM, part 2: complicated grief. J Clin Psychiatry 2010;71:1097-8.

14 Prigerson HG, Horowitz MJ, Jacobs SC, et al. Prolonged grief disorder: psychometric validation of criteria proposed for DSM-V and ICD-11. PLoS Med 2009;6:e1000121.

15 Hutti MH, Armstrong DS, Myers JA, et al. Grief intensity, psychological well-being, and the intimate partner relationship in the subsequent pregnancy after a perinatal loss. J Obstet Gynecol Neonatal Nurs 2015;44:42-50.

16 O'Connor M, Nickerson A, Aderka IM, et al. The temporal relationship between change in symptoms of prolonged grief and posttraumatic stress following old age spousal bereavement. Depress Anxiety 2015;32:335-40.

17 Muñoz RF. Using evidence-based Internet interventions to reduce health disparities worldwide. J Med Internet Res 2010;12:e60.

18 Boelen PA, van de Schoot R, van den Hout MA, et al. Prolonged grief disorder, depression, and posttraumatic stress disorder are distinguishable syndromes. J Affect Disord 2010;125:374-8.

19 Wagner B, Knaevelsrud C, Maercker A. Internet-Based cognitivebehavioral therapy for complicated grief: a randomized controlled trial. Death Stud 2006;30:429-53.

20 van der Houwen $\mathrm{K}$, Schut $\mathrm{H}$, van den Bout J, et al. The efficacy of a brief Internet-based self-help intervention for the bereaved. Behav Res Ther 2010;48:359-67.

21 Kleinman A, Culture KA. Culture, bereavement, and psychiatry. Lancet 2012;379:608-9.

22 Schut H, Stroebe MS, Van den Bout J. The efficacy of bereavement interventions: determining who benefits. In: Handbook of bereavement research: consequences, coping, and care. Washington DC, US: American Psychological Association, 2001: 705-37.

23 Kersting A, Dölemeyer R, Steinig J, et al. Brief Internet-based intervention reduces posttraumatic stress and prolonged grief in parents after the loss of a child during pregnancy: a randomized controlled trial. Psychother Psychosom 2013;82:372-81.
24 Moher D, Liberati A, Tetzlaff J, et al. Preferred reporting items for systematic reviews and meta-analyses: the PRISMA statement. BMJ 2009;339:b2535.

25 Shamseer L, Moher D, Clarke M, et al. Preferred reporting items for systematic review and meta-analysis protocols (PRISMA-P) 2015 elaboration and explanation. BMJ 2015;350:g7647.

26 Kampling $\mathrm{H}$, Baumeister $\mathrm{H}$, Jaeckel $\mathrm{WH}$, et al. Prevention of depression in chronically physically ill adults. Cochrane Database Syst Rev 2014:CD011246.

27 Higgins JPT, Altman DG, Gøtzsche PC, et al. The Cochrane collaboration's tool for assessing risk of bias in randomised trials. BMJ 2011;343:d5928.

28 Guyatt GH, Oxman AD, Vist GE, et al. Grade: an emerging consensus on rating quality of evidence and strength of recommendations. $B M J$ 2008;336:924-6.

29 Schünemann H, Brożek J, Guyatt G, et al. GRADEpro GDT. GRADE Handbook, 2013. Available: https://gdt.gradepro.org/app/handbook/ handbook.html\#h.svwngs6pm0f2

30 Valentine JC, Pigott TD, Rothstein HR. How many studies do you need? A primer on statistical power for meta-analysis. J Educ Behav Stat 2010;35:215-47.

31 Gerhards SAH, de Graaf LE, Jacobs LE, et al. Economic evaluation of online computerised cognitive-behavioural therapy without support for depression in primary care: randomised trial. $\mathrm{Br} \mathrm{J}$ Psychiatry 2010;196:310-8.

32 Hedman E, Andersson E, Lindefors N, et al. Cost-Effectiveness and long-term effectiveness of Internet-based cognitive behaviour therapy for severe health anxiety. Psychol Med 2013;43:363-74.

33 Hedman E, Andersson E, Ljótsson B, et al. Cost-Effectiveness of Internet-based cognitive behavior therapy vs. cognitive behavioral group therapy for social anxiety disorder: results from a randomized controlled trial. Behav Res Ther 2011;49:729-36.

34 Kersting A, Brähler E, Glaesmer $\mathrm{H}$, et al. Prevalence of complicated grief in a representative population-based sample. J Affect Disord 2011;131:339-43. 